\title{
FOURIER TRANSFORMS OF LIPSCHITZ FUNCTIONS ON CERTAIN LIE GROUPS
}

\author{
M. S. YOUNIS
}

(Received 27 March 2000)

\begin{abstract}
We study the order of magnitude of the Fourier transforms of certain Lipschitz functions on the special linear group of real matrices of order two.
\end{abstract}

2000 Mathematics Subject Classification. 42A38, 44A15, 42C99, 44A05.

1. Introduction. The order of magnitude of the Fourier transforms (coefficients) of Lipschitz functions on various domains is an active field of investigation. For example in $[9,11]$ this problem was studied for Lipschitz functions on the groups SU(2) and $\mathrm{SU}(1,1)$. In the present work we take the same problem for Lipschitz functions but defined on $\operatorname{SL}(2, \mathbb{R})$, the special linear group of real matrices of order two. This note is organized as follows: in Section 2, we give the necessary definitions and notation to be employed in the sequel. In Section 3, we deal with the problem in the context of $L^{2}$, the space of square integrable functions on $\operatorname{SL}(2, \mathbb{R})$. The main conclusions obtained are extended to functions in $L^{p}, 1<p \leq 2$ in Section 4 . The closing Section 5 is devoted to few remarks and comments pointing to possible further extensions.

2. Definitions and notation. We collect here the basic material needed for our work in the sequel. In this note $G$ stands for the group $\operatorname{SL}(2, \mathbb{R})$ unless mentioned otherwise. Our main sources of information on the group $G$ are [3, 5]. The treatment of the subject matter is based on a suitable decomposition of $G$. Several decompositions of $G$ are available in the literature (see [3, page 199] and [5, page 351]). In particular, there is the Iwasawa decomposition given as $G=K A N$, where

$$
\begin{aligned}
K & =\left[\begin{array}{cc}
\cos \theta & \sin \theta \\
-\sin \theta & \cos \theta
\end{array}\right], \quad 0<\theta<2 \pi \\
A & =\left[\begin{array}{cc}
e^{\phi} & 0 \\
0 & e^{-\phi}
\end{array}\right], \quad 0<\phi<\pi \\
N & =\left[\begin{array}{ll}
1 & t \\
0 & 1
\end{array}\right], \quad t \in \mathbb{R} .
\end{aligned}
$$

Two other decompositions are $G=K_{1} A K_{2}$ and $G=A_{1} K A_{2}$, where $K_{1}, K_{2}, A_{1}$, and $A_{2}$ are similar copies of $K$ and $A$. The domain of parameters used in these decompositions will be specified in due course. We emphasize here that the variety of decompositions of $G$ do not cause any annoyance to us, they give rise to different but equivalent Haar 
measures on $G$. Thus in the form $G=K A N$ if $f(g) \in L^{1}(G), g \in G$, then

$$
\int_{G} f(g) d g=\int_{0}^{2 \pi} \int_{0}^{\infty} \int_{0}^{\infty} f\left(u_{\phi}, a_{t}, n_{\xi}\right) e^{t} d \phi d t d \xi
$$

where $u_{\phi} \in K, a_{t} \in A$, and $n_{\xi} \in N$, whereas if $G=K_{1} A K_{2}$, then (2.2) takes the form

$$
\int_{G} f(g) d g=\int_{0}^{2 \pi} \int_{0}^{\infty} \int_{0}^{2 \pi} f(g) \sinh t d \phi d t d \psi,
$$

here $f(g)=f\left(u_{\phi}, a_{t}, u_{\psi}\right)$, (see [3, pages 251-252]).

We have dropped the normalizing factors from the integrals, their presence is not necessary.

It is noted that in the second decomposition one domain $[(0, \infty)]$ is suppressed into $[0,2 \pi]$. This is immaterial here since the real line $\mathbb{R}$ is the product of the circle group $T$ and the group of integers $\mathbb{Z}$ which in turn has a compact dual (on which our conclusions hold trivially) isomorphic to $T$ according to the famous duality of topological groups (see [1, Theorem 24.8, page 378]). So, it is enough to check the validity of a certain conclusion for functions defined on the circle group $T$ in order to be convinced that it holds for functions defined on the real line. The interested reader may consult $[6,7]$ to see that the theorems proved for Lipschitz functions on the circle group (on the torus $T^{n}$ in general) have exactly the same conclusions as the theorems proved for Lipschitz functions on $\mathbb{R}$ (on the $n$-dimensional Euclidean space $\mathbb{R}^{n}$ in general). It should be stressed, however, that one cannot suppress the two infinite domains $(0, \infty)$ altogether, simply because $G$ is noncompact (locally compact). If one is obliged to do so, he must employ the structure theorem of topological groups for instance. In the present work we take the decomposition $G=A_{1} H A_{2}$, where $A_{1}$ and $A_{2}$ are diagonal groups with parameters $\phi, \psi, 0 \leq \phi, \psi \leq 2 \pi$ and $H=H(\theta)=$ $\left[\begin{array}{c}\cosh \theta \sinh \theta \\ \sinh \theta\end{array} \cosh \theta\right],-\infty<\theta<\infty$, (see [5, pages 351, 364]). In this decomposition we discarded matrices of the forms $\left[\begin{array}{cc}-1 & 0 \\ 0 & -1\end{array}\right]$ and $\left[\begin{array}{cc}0 & 1 \\ -1 & 0\end{array}\right]$ since their presence has no significant bearing on the present work. With these (so-called Euler) parameters the Haar integral on $G$ takes the form in (2.3).

Coming now to Lipschitz functions we recall that if $f=f(x, y, z) \in L^{p}\left(\mathbb{R}^{3}\right)\left(L^{p}\left(T^{3}\right)\right)$, then $f$ is said to belong to the Lipschitz class Lip $\left(\alpha_{1}, \alpha_{2}, \alpha_{3}, p\right), 1<p \leq 2,0<\alpha_{i} \leq 1$, if

$$
\left\|\Delta_{h_{1}} \Delta_{h_{2}} \Delta_{h_{3}} f\right\|_{p}=O\left(h_{1}^{\alpha_{1}} h_{2}^{\alpha_{2}} h_{3}^{\alpha_{3}}\right)
$$

as $h_{i} \rightarrow 0, i=1,2,3$, where $\Delta_{h_{1}} \Delta_{h_{2}} \Delta_{h_{3}} f$ stands for the successive differences of $f$ with steps $h_{1}, h_{2}$, and $h_{3}$ in $x, y$, and $z$, respectively, $\|\cdot\|_{p}$ is the usual $L^{p}$ norm. As was shown in $[6,7]$. This definition is equivalent to another one in which we take $h_{i}=h$, and $0<\alpha=\alpha_{1}+\alpha_{2}+\alpha_{3} \leq 3$. Thus (2.4) could be written as

$$
\left\|\Delta_{h} \Delta_{h} \Delta_{h} f\right\|_{p}=O\left(h^{\alpha}\right)
$$

Besides its brevity the expression in (2.5) will facilitate the writing of the proof considerably as will be seen. We start with the following definition. 
DEFINITION 2.1. Let $f(g)=f(\phi, \theta, \psi) \in L^{p}(G), 1<p \leq 2$. Then $f \in \operatorname{Lip}(\alpha, p, G)$ if (2.5) holds as $h \rightarrow 0,0<\alpha \leq 3$.

One could still cast Definition 2.1 for increments $h_{i}$, with $0<\alpha_{i} \leq 1, i=1,2,3$ as in (2.4) if necessary. In order to see the type of conclusions if (2.4) were to be employed, the reader may refer to $[6,7]$ where he can find detailed analysis in connection with the two alternatives (2.4) and (2.5).

Our next target in this section is to choose a suitable form of the group representation. Two basic forms are to be found in [3, pages 208-209] and in [5, page 359]. Thus for a function $f(g), g \in G$ the representation in [3] is given by

$$
\left(V_{g}^{j, s} f\right)(\xi)=|\bar{\beta} \xi+\bar{\alpha}|^{-2 s}\left(\frac{\bar{\beta} \xi+\bar{\alpha}}{|\bar{\beta} \xi+\bar{\alpha}|}\right)^{2 j} f\left(g^{-1} \cdot \xi\right),
$$

where $g^{-1} \cdot \xi=(\alpha \xi+\beta) /(\bar{\beta} \xi+\bar{\alpha})$,

$$
j=0, \frac{1}{2}, \quad s=\frac{1}{2}+i \sigma, \quad \sigma \in \mathbb{R} .
$$

In [5, page 359] one finds the following

$$
T_{X}(g) f(x)=|\beta x+\delta|^{2 l} \operatorname{sign}(\beta x+\delta)^{2 \varepsilon} f\left(\frac{\alpha x+\gamma}{\beta x+\delta}\right),
$$

where $\chi=(l, \varepsilon), l=1 / 2+i \lambda, \lambda \in \mathbb{R}, \varepsilon=0,1 / 2$.

Taking into account the way in which the matrix elements $g$ and $g^{-1}$ are defined and comparing parameters carefully, one concludes that the two forms (2.6) and (2.8) are exactly the same. In addition to (2.8) Vilenkin (see [5, page 361]) gives another (yet equivalent) form of $T_{X}(g) f$ in terms of the Mellin transform $F(\lambda)$ of $f(x)$ (see [5, pages 356-357]), for the definition of $F(\lambda)$ for $x>0, x<0$, and for inversion formula of the Mellin transform. Thus one has the following

$$
T_{X}(g) f(x)=\mathbb{R}_{X}(g) F(\lambda)=\mathbb{R}_{X}(g)\left(F_{+}(\lambda), F_{-}(\lambda)\right)=\int_{a-i \infty}^{a+i \infty} K(\lambda, \mu, g) F(\mu) d \mu,
$$

where $K$ (called by Vilenkin the kernel of the group representation) is written as

$$
K=\left[\begin{array}{ll}
K_{++} & K_{+-} \\
K_{-+} & K_{--}
\end{array}\right] .
$$

In this formulation $\mathbb{R}_{X}(g) F_{+}$is expressed in terms of two integrals containing $K_{++}$, $K_{+-}$, whereas $\mathbb{R}_{X}(g) F_{-}$is given by two integrals containing $K_{-+}$and $K_{--}$. A typical component $K_{++}$of the kernel is given by (see [5, page 361])

$$
K_{++}(\lambda, \mu, g)=\int_{0}^{\infty} x^{\lambda-1}\left|\frac{\alpha x+\gamma}{\beta x+\delta}\right|_{+}^{-\mu}|\beta x+\delta|^{2 l} \operatorname{sign}^{2 \varepsilon}|\beta x+\delta| d x .
$$

Since the three other components of $K$ are defined basically by (2.11) with slight changes in the sign of the variable $x$ (which are immaterial in the present work), 
it turns out that if we succeed in solving our problem for $K_{++}$, then virtually it is completely solved taking into account the decomposition $G=A_{1} H A_{2}$ and the fact that for both $A_{1}(\phi)$ and $A_{2}(\psi)$ the representations are given by $e^{i m \phi}$ and $e^{i n \psi} m, n \in \mathbb{Z}$, respectively, we arrive finally at the cornerstone of the present analysis by tackling our problem on the kernel $K_{++}$of $\mathbb{R}_{X}(g) F_{+}$which we denote by $\mathbb{R}_{+\chi}(g) F_{+}$for brevity, viz.

$$
\mathbb{R}_{+_{\chi}}(g) F_{+}=e^{i(m \phi+n \psi)} \int_{0}^{\infty} K_{++}(\lambda, \mu, g) F(\mu) d \mu .
$$

It is (almost entirely) on this component that our analysis will be based. Thus, in view of what has been already achieved, the only problem to be tackled is the effect of Lipschitz conditions in the variable $\theta$ on the order of magnitude of the Fourier transforms of functions in $L^{p}(G)$. In $[9,11]$ we treated similar situations by proving a lemma as a prelude to the main theorems. This is what we are going to do in the next section also.

3. Main theorems. For brevity the Fourier transform of $f$ will be denoted by $\hat{f}$. As we mentioned earlier, we focus our attention on $f(g)=f(\phi, \theta, \psi)$ as a function of $\theta$, since in terms of the variables $\phi$ and $\psi$ the problem reduces simply to that of functions of two variables in $L^{p}\left(T^{2}\right)$ or in $L^{p}\left(\mathbb{R}^{2}\right)$ and this has been already settled (see [6, 7], for example). The Fourier transform of $f(g) \in L^{1}(G)$ is given as (see [3, page 329])

$$
\begin{aligned}
\hat{f}(j, s) & =\int_{G} f(g) V_{g^{-1}}^{j, s} d g, \\
\hat{f}(n) & =\int_{G} f(g) U_{g^{-1}}^{n} d g .
\end{aligned}
$$

Here (3.1) and (3.2) give the contributions to $\hat{f}$ by the principal continuous series and the discrete series of the representation (no contribution by the complementary continuous series). We will leave (3.2) aside for a while and will return to it after we have finished with (3.1). Firstly, taking into consideration the decomposition $G=$ $A_{1}(\phi) H(\theta) A_{2}(\psi)$ and viewing $f(g)$ as a function of $\theta$, we can write (3.1) as follows:

$$
\hat{f}(j, s)=\hat{f}(\varepsilon, l)=\int_{0}^{\infty} f(\theta)\left(\int_{0}^{\infty} K_{++}(\lambda, \mu, H(\theta)) F(\mu) d \mu\right) \sinh \theta d \theta .
$$

Turning to $K_{++}$and replacing $\alpha, \beta, \gamma$, and $\delta$ in (2.11) by their corresponding parameters in $H(\theta)$ we see that (see [5, page 365, equation (3)])

$$
K_{++}=\int_{0}^{\infty} x^{\lambda-1}(x \cosh \theta+\sinh \theta)^{-\mu}(x \sinh \theta+\cosh \theta)^{2 l+\mu} d x .
$$

It is obvious that as a function of $\theta, K_{++}=O(\cosh \theta)^{2 l}$. This can also be seen easily from (2.11), since the only part (in the integral) which contributes to this order of magnitude is $|\beta x+\delta|^{2 l}$. We emphasize that this estimate for $K_{++}$is shared by all the other components of the kernel $K$. Strictly speaking, if we are able to settle our problem for $K_{++}$, then we are completely done with the whole issue. Observe that one could have as well estimated $K_{++}$by $O(\sinh \theta)^{2 l}$, but this would create unnecessary difficulties 
( $\operatorname{coth} \theta$ goes to infinity as $\theta$ approaches zero). We now take $K_{++}=B(\cosh \theta)=B(y)$ for brevity and proceed by proving the following lemma.

LEMMA 3.1. Let $f(g)=f(\cdot, \theta, \cdot)$ belong to $L^{1}(G)$. Then $\left|\Delta_{h} \hat{f}\right|=\mid f(\theta+h)-f(\hat{\theta})=$ $O(h \hat{f})$.

Proof. With the new notation for $K_{++}$, the transform of $\Delta_{h} f(\theta)$ is

$$
\int_{0}^{\infty}|f(\theta+h)-f(\theta)| B(\cosh \theta) \sinh \theta d \theta
$$

by a slight change of variables (3.5) is equal to

$$
\begin{aligned}
\int_{1}^{\infty} \mid & f(y+h)-f(y) \mid B(y) d y \\
& =\int_{1}^{\infty}|f(y)|(B(y-h)-B(y)) d y \\
& =O \int_{1}^{\infty}|f(y)|\left((y-h)^{2 l}-y^{2 l}\right) d y \\
& =O \int_{1}^{\infty}|f(y)| y^{2 l}\left(\left(1-\frac{h}{y}\right)^{2 l}-1\right) d y .
\end{aligned}
$$

Since $(1-h / y)^{2 l}-1=1-(2 l h / y)-1+$ terms of higher order in $h$ which can be neglected, and since $1 / y$ is bounded near 1 and $\infty$, the last integral is majorized by a constant multiple of the integral

$$
h \int_{1}^{\infty} f(y) B(y) d y=O(h \hat{f}) .
$$

This proves the lemma. At this point it should be recalled that $\hat{f}(j, s)=\hat{f}(\varepsilon, l)$ given by (3.3) splits actually into two integrals corresponding to $\varepsilon=0$, and $\varepsilon=1 / 2$, respectively. A careful examination of the definition of $K_{++}$as well as the proof of Lemma 3.1 shows that these two values of $\varepsilon$ have no bearing on the main lines of the proof. In view of this we can simplify our notation by discarding the parameter $\varepsilon$ and writing $\hat{f}=\hat{f}(l)$ which can be expressed as follows

$$
\hat{f}(l)=\int_{0}^{2 \pi} \int_{0}^{\infty} \int_{0}^{2 \pi} f(\phi, \theta, \psi) e^{i(m \phi+n \psi)}\left[\int_{0}^{\infty} K_{++} F(\psi) d \mu\right] \sinh \theta d \phi d \theta d \psi .
$$

Integrals corresponding to $K_{+-}, \ldots$ can be expressed in a similar fashion.

We are now ready to prove the following theorem.

THEOREM 3.2. Let $f(g)$ belong to $L^{2}(G)$ such that

$$
\left\|\Delta_{h} \Delta_{h} \Delta_{h} f\right\|_{2}=O\left(h^{\alpha}\right), \quad \frac{1}{2}<\alpha \leq 3, h \rightarrow 0 .
$$

Then

$$
\int_{\lambda}^{\infty}\|\hat{f}(1)\|_{2}^{2} \lambda \tan h \pi \lambda d \lambda=O\left(\lambda^{-2 \alpha}\right)
$$

as $\lambda \rightarrow \infty$ and conversely. 
Proof. It is well known (see [6, 7], for example) that the Lipschitz conditions on $f$ with respect to $\phi$ and $\psi$ result in the multiplicative factor

$$
e^{i j(m \phi+n \psi) / 2} \sin \frac{m h}{2} \sin \frac{n h}{2}
$$

which has a modulus of the order of $\left|m n h^{2}\right|, 0<m, n<1 / h$. The effect of Lipschitz condition in $\theta$ is given by Lemma 3.1. Putting all these results together one finds that $\left|\Delta_{h} \Delta_{h} \Delta_{h} \hat{f}\right|=O\left|m n h^{3} \hat{f}\right|$. Applying the Parseval's identity (see [3, page 346]) we obtain

$$
\begin{gathered}
\int_{0}^{\infty} \sum_{m=1}^{M} \sum_{n=1}^{N}\left|m n h^{3} \hat{f}\right|^{2} \lambda \tan h \pi \lambda d \lambda=O\left(h^{2 \alpha}\right), \\
\int_{0}^{\infty} \sum_{1}^{M} \sum_{1}^{N}|m n \hat{f}|^{2} \lambda \tan h \pi \lambda d \lambda=O\left(h^{2 \alpha-6}\right) .
\end{gathered}
$$

Recalling that $\tanh \pi \lambda \rightarrow 1$ as $\lambda \rightarrow \infty$ and appealing to the proof of [4, Theorem 85 , page 117] (see also [6, 7]), with the observation that $m, n$, and $\lambda$ approach infinity at the same rate we conclude that

$$
\int_{X}^{\infty} \sum_{m>|X|}^{\infty} \sum_{n>|X|}^{\infty}|m n \hat{f}|^{2} \lambda \tan h \pi \lambda d \lambda=O\left(X^{6-2 \alpha}\right)
$$

or equivalently (by a straightforward partial summation argument)

$$
\int_{X}^{\infty} \sum_{X}^{\infty} \sum_{X}^{\infty}|\hat{f}|^{2} \lambda d \lambda=O\left(X^{2-2 \alpha}\right), \quad \int_{X}^{\infty} \sum_{X}^{\infty} \sum_{X}^{\infty}|\hat{f}|^{2} d \lambda=O\left(X^{1-2 \alpha}\right)
$$

as $X \rightarrow \infty$. The condition $\alpha>1 / 2$ gives the convergence of the left-hand side of (3.14), and thus the first part of the theorem is proved. To prove the converse one could resort to the corresponding part in [5, page 117] and in more details to [6, 7], this part of the proof will not be given here.

It must have been clear by now that one can obtain the same result for the second component of $\hat{f}$ given by (3.2), namely $\hat{f}(n)$ by just replacing integration in (3.14) by yet another summation and tackling the relevant part of the Parseval's identity. Thus by these remarks and illustrations the theorem is valid for the three components of $\hat{f}$. This completes the proof. In fact, one can go few steps forward. Firstly, since (3.14) applies to each component of $\hat{f}$, it applies to their sum also in the sense of the Parseval's identity. Another point is that because $K_{-+}, K_{+-}, K_{--}$are either zero (see [5, page 365]) or have similar structures of $K_{++}$, one can obtain in each individual case exactly the same conclusions already obtained for $K_{++}$, thus proving the theorem in its full generality.

We like to indicate that whether one works with $\mathbb{R}_{X}(g)$ or with $T_{X}(g)$ the conclusion of Theorem 3.2 is the same, simply because in both cases the Lipschitz conditions in $\phi, \theta$, and $\psi$ have not much to do with other parameters, however, we feel that $\mathbb{R}_{X}$ is more compact and lends itself easily to the present analysis more than $T_{X}$. This 
easiness comes mainly from the role of the Mellin transform in $\mathbb{R}_{X}$. Another point is that one could have easily applied (2.4) in the proof; eventually this would have given estimates of the form

$$
\begin{aligned}
\int_{X}^{\infty} \sum_{M}^{\infty} \sum_{N}^{\infty}|\hat{f}|^{2} \lambda d \lambda & =O\left(M^{-2 \alpha_{1}} N^{-2 \alpha_{2}} X^{2-2 \alpha_{3}}\right), \\
\int_{X}^{\infty} \sum_{M}^{\infty} \sum_{N}^{\infty}|\hat{f}|^{2} d \lambda & =O\left(M^{-2 \alpha_{1}} N^{-2 \alpha_{2}} X^{2-2 \alpha_{3}}\right),
\end{aligned}
$$

as $M, N$, and $X \rightarrow \infty$, along with the condition $\alpha_{3}>1, \alpha_{3}>1 / 2$ for the boundedness of the left-hand sides of (3.15), respectively. The proof in this case can be carried out with no difficulty, except for some technical complications.

REMARK 3.3. We hinted earlier that Lipschitz functions in $L^{p}\left(T^{n}\right)$ and in $L^{\mathbb{R}}\left(\mathbb{R}^{n}\right)$ yield the same conditions for the boundedness of their Fourier coefficients (transforms) in certain function spaces, we explained that on the grounds of duality and structure theorems for locally compact groups. Here we meet a similar situation in the sense that for the Lipschitz functions on $\operatorname{SU}(2)$, on $\operatorname{SU}(1,1)$, and on $\operatorname{SL}(2, \mathbb{R})$ the conditions as well as the conclusions of the main theorems are the same apart from the increasing generality from Jacobi polynomials on SU(2), through the Jacobi functions on $\operatorname{SU}(1,1)$ to the hypergeometric functions on $\operatorname{SL}(2, \mathbb{R})$. This is not surprising since, in the integral representation of these three functions, it is the $|B x+\delta|^{2 l}$ which plays the essential role in connection with the effect of Lipschitz conditions on the order of magnitude of the Fourier transforms. For more on the integral representations of these functions one may consult [5, Chapters 3, 6, 7]. We remark here that in $[9,11]$ both the Jacobi polynomials and functions appeared in our estimates, whereas in here we have deliberately avoided the use of the hypergeometric functions and preferred (for ease and smoothness of some expressions) to work directly with $K_{++}$. For rather complicated relations between the various components $K_{++}, K_{+-}, \ldots$ and hypergeometric functions the interested reader may refer to [5, page 365].

REMARK 3.4. So far we have worked with a decomposition of $G$ characterized by the Euler angles as the group parameters. The natural question arises as to the capability of carrying the present analysis on the Iwasawa decomposition: $G=K A N$. We do conjecture that the answer will be in the affirmative. We support this by emphasizing that on the subgroup $A$ the problem boils down to the order of magnitude of the Mellin transforms of Lipschitz functions, a problem which was studied in [8]. On the subgroup $N$ the question reduces to the Radon transforms of certain Lipschitz classes. The close relation between these two transforms and the ordinary Fourier transform would enhance our surmise that in the Iwasawa decomposition of $G$ the above analysis is quite amenable to be fully implemented without difficulty.

4. Further extensions. In this section we try to extend Theorem 3.2 to Lipschitz functions in $L^{p}(G), 1<p \leq 2$. Here one invokes the Hausdorff-Young inequality instead of the Parseval's identity. In contrast with the $L^{2}$ theory, our problem is not reversible in $L^{p}(G)$. Now we state the following theorem. 
THEOREM 4.1. Let $f(g)$ belong to $L^{p}(G), 1<p \leq 2$, such that (2.5) is satisfied for $1 / p<\alpha \leq 3$. Then each component of $\hat{f}$ belongs to $L^{r}(\hat{G})$ for

$$
3 p(\alpha p+3 p-4)<r \leq p^{\prime}=\frac{p}{(p-1)},
$$

where $\hat{G}$ is the group dual to $G$.

The proof will not be given in detail; it is modeled on that of Theorem 3.2 the main equations in the argument are

$$
\begin{aligned}
& \int_{0}^{\infty} \sum_{1}^{\infty} \sum_{1}^{\infty}\left|m n h^{3} \hat{f}\right|^{p^{\prime}} \lambda \tan h \pi \lambda d \lambda=O\left(h^{\alpha p^{\prime}}\right) \\
& \int_{0}^{\lambda} \sum_{1}^{M} \sum_{1}^{N}|m n \hat{f}|^{p^{\prime}} \lambda \tan h \pi \lambda d \lambda=O\left(h^{\alpha p^{\prime}-3 p^{\prime}}\right)=O\left(X^{3 p^{\prime}-\alpha p^{\prime}}\right) \\
& \int_{0}^{\lambda} \sum_{1}^{M} \sum_{1}^{N}|\hat{f}|^{p^{\prime}} \lambda \tan h \pi \lambda d \lambda=O\left(X^{p^{\prime}-\alpha p^{\prime}}\right)
\end{aligned}
$$

or equivalently

$$
\int_{0}^{\lambda} \sum_{1}^{M} \sum_{1}^{N}|\hat{f}|^{p^{\prime}} d \lambda=O\left(X^{p^{\prime}-\alpha p^{\prime}-1}\right),
$$

where $M, N$, and $\lambda=O(X)$ near infinity.

Hölder's inequality when applied to (4.2) and (4.3) for $r<p^{\prime}$ yields the conditions, $4 p /(\alpha p+3 p-4)<r \leq p^{\prime}, 3 p /(\alpha p+3 p-4)<r \leq p^{\prime}$, respectively, for the boundedness of the last estimates, this completes the proof. We close this section by indicating that most of the comments and ramifications mentioned in the last section are valid here too with the necessary modifications and therefore will not be repeated again.

5. Concluding remarks. We first hint that in view of their generality, the theorems proved in the present work embrace all those already worked out in [9, 11]. This is simply because the representation (especially their matrix elements) of SU(2) and $\mathrm{SU}(1,1)$ are just special cases of those for $\operatorname{SL}(2, \mathbb{R})$. In addition, the present analysis is applicable for the Lorentz group $G(2)$ without much effort (see [3, page 205]) for the relation between $G(2)$ and $\operatorname{SL}(2, \mathbb{R})$.

Since the kernel $\phi(g, s)$ (see [3, page 349]) of the spherical Fourier transform on $\mathrm{SL}(2, \mathbb{R})(\mathrm{SU}(1,1))$ is a special form of the matrix element $T_{X}(g)$ (in fact it is a special form of the $\left.K_{++}, \ldots\right)$, hence the order of magnitude of the spherical-Fourier transforms of Lipschitz functions is automatically included in the above results. We shall not deal with that question here. For more information in this direction one may consult [10], where the spherical-Fourier transforms of Lipschitz classes on the hyperbolic plane (which is isomorphic to the unit disc with its Riemannian structure as a homogeneous space associated with the group $\mathrm{SU}(1,1))$ is studied.

The rich variety of the special subgroups of $G$ such as those given by $\left[\begin{array}{ll}\alpha & B \\ 0 & 8\end{array}\right],\left[\begin{array}{ll}1 & B \\ 0 & 1\end{array}\right]$, $\left[\begin{array}{cc}\alpha & 0 \\ \gamma & \delta\end{array}\right]$, and so forth, leads to numerous classes of functions along with some of their integral representations (transforms). The Mellin, the Radon, and the Mehler-Fock 
transforms are just few examples. See [5, page 541] for the definition of the MehlerFock transforms.

It is well known that for smooth functions defined on non-abelian groups (cf. [2]) there are several criteria for the absolute convergence (and for the order of magnitude) of their Fourier transforms (coefficients). In [11], we worked out some of these criteria for the Jacobi polynomials. Here, one could do the same thing for the various components of $\hat{f}$, however, we will not go into these issues here, one may refer to [11] for more on this topic.

We have employed here the rather conventional Lipschitz class $\operatorname{Lip}(\alpha, p)$. For more general spaces (Lorentz, Besov, Nikolski, and Herz, to mention a few) the present treatment would lead to interesting results. This task needs a lot of preparation and will be studied in a forthcoming paper. Still, a more formidable and overwhelming task lies ahead; the extension of our conclusions to Lipschitz functions on more general groups $(\mathrm{SU}(m, n), \mathrm{SO}(n), \mathrm{SP}(p, q)$, and semi-simple Lie groups) and on $\operatorname{SL}(n, \mathbb{R})$ in particular. In the Iwasawa decomposition $K A N$ of this group the analysis on $A$ would amount to an $n$-dimensional Mellin transform, a problem which is easily manageable within the framework of the above analysis. On $K$ and $N$, however, things seem to be rather vague, especially the structure of the kernel of representation $K$ and its components in case of an $n \times n$ matrix with trigonometric or hyperbolic entries. One has a strong feeling that this would lead to a higher order of complexity (generalized hypergeometric functions and the so called ultra spherical functions) in the functions treated on $\operatorname{SL}(2, \mathbb{R})$.

Although a decomposition of $\operatorname{SL}(n, \mathbb{R})$ amenable to the usage of Euler parameters would make the problem more akin to an easier approach, where in that decomposition the elements of the representations corresponding to the $\phi$ 's and $\psi$ 's are simply those obtained for the Euclidean Fourier analysis (exponential functions of several variables). There still remains the main hurdle (bête noire) of finding the kernel $K$ along with its nonvanishing components. We hope to have some progress on these issues along with the treatment of the present subject for Lipschitz functions on $M H(2)$, the group of motions in the pseudo Euclidean plane and $M H(n)$, the group of hyperbolic rotations of the $n$-dimensional Euclidean space (see $[5$, Chapters $5,8,10]$ ).

ACKNOWLEDGEMENTS. This work was prepared while the author was on a sabbatical leave from Yarmouk University as a research scholar at the Department of Mathematics in the University of British Colombia.

This work is dedicated to Prof M. Hailat, a colleague and a friend with whom the topic of Lie Algebra has always been a source of real enjoyment and fruitful information.

\section{REFERENCES}

[1] E. Hewitt and K. A. Ross, Abstract Harmonic Analysis. Vol. I: Structure of Topological Groups. Integration Theory, Group Representations, Die Grundlehren der mathematischen Wissenschaften, vol. 115, Springer-Verlag, New York, 1963. MR 28\#158. Zbl 115.10603.

[2] D. L. Ragozin, Approximation theory, absolute convergence, and smoothness of random Fourier series on compact Lie groups, Math. Ann. 219 (1976), no. 1, 1-11. MR 53\#13961. Zbl 307.43012. 
[3] M. Sugiura, Unitary Representations and Harmonic Analysis. An Introduction, Kodansha, Tokyo, 1975. MR 58\#16977. Zbl 344.22001.

[4] E. C. Titchmarsh, Theory of Fourier Integrals, 2nd ed., Oxford University Press, London, 1948.

[5] N. J. Vilenkin, Special Functions and the Theory of Group Representations, Translations of Mathematical Monographs, vol. 22, American Mathematical Society, Rhode Island, 1968. MR 37\#5429. Zbl 172.18404.

[6] M. S. Younis, Fourier transforms of Dini-Lipschitz functions, Int. J. Math. Math. Sci. 9 (1986), no. 2, 301-312. MR 88b:42019. Zbl 595.42006.

[7] _ Fourier transformations of functions with symmetrical differences, Acta Math. Hungar. 51 (1988), no. 3-4, 293-299. MR 89k:42009. Zbl 672.42005.

[8] _ The Fourier transforms of Lipschitz functions on certain domains, Int. J. Math. Math. Sci. 20 (1997), no. 4, 817-822. CMP 1490 742. Zbl 912.42007.

[9] _ The Jacobi Fourier transforms of certain Lipschitz functions on SU (1, 1), Math. Sci. Res. Hot-Line 1 (1997), no. 11, 15-24. MR 99i:44008. Zbl 960.44500.

[10] _ Fourier transforms of Lipschitz functions on the hyperbolic plane $H^{2}$, Int. J. Math. Math. Sci. 21 (1998), no. 2, 397-401. MR 99c:43018. Zbl 903.42002.

[11] _ On the Jacobi-Fourier transforms of certain Lipschitz functions, J. Inst. Math. Comput. Sci. Math. Ser. 13 (2000), no. 3, 333-338. CMP 1815323.

M. S. YOUNIS: DEPARTMENT OF MATHEMATICS, YARMOUK UNIVERSITY, IRBID, JORDAN

E-mail address: younis@yu.edu.jo 


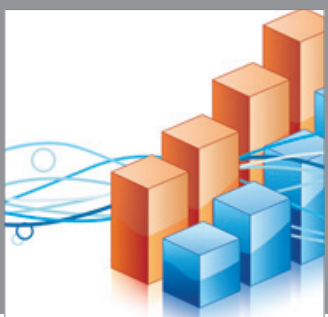

Advances in

Operations Research

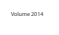

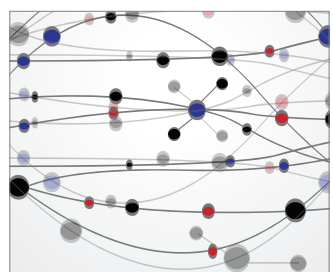

\section{The Scientific} World Journal
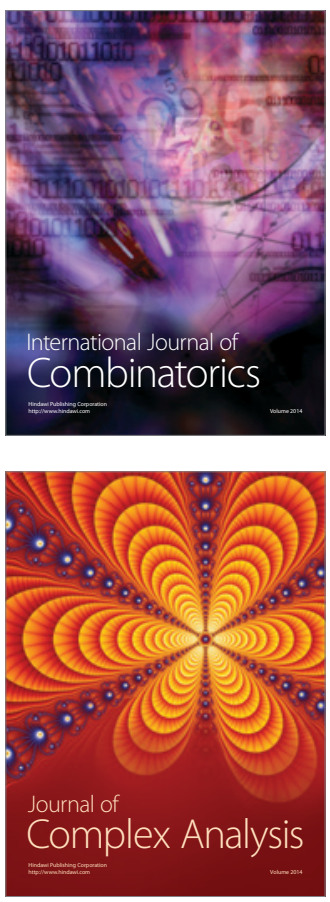

International Journal of

Mathematics and

Mathematical

Sciences
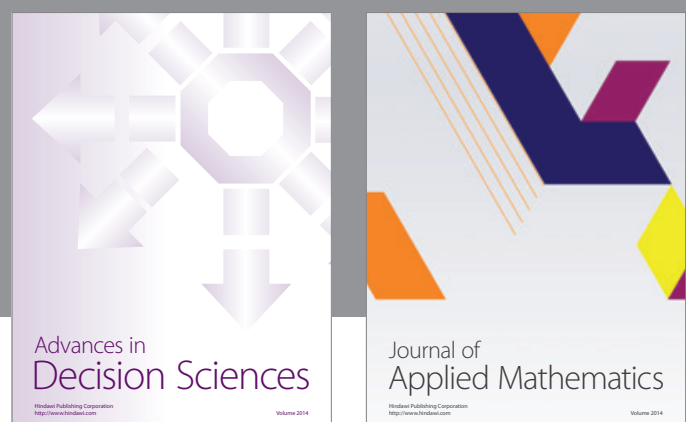

Journal of

Applied Mathematics
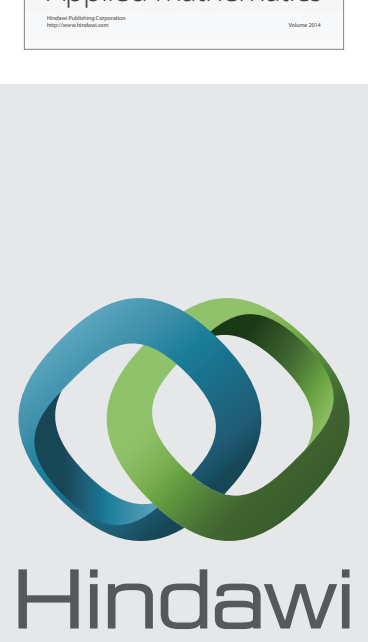

Submit your manuscripts at http://www.hindawi.com
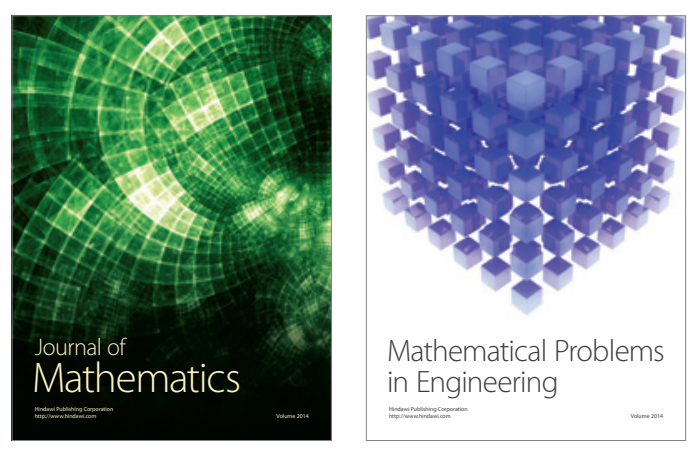

Mathematical Problems in Engineering
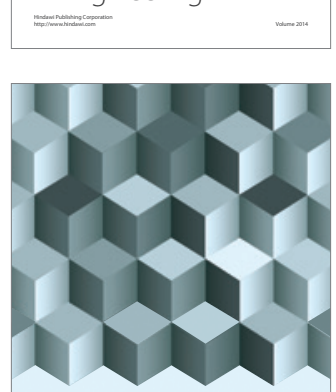

Journal of

Function Spaces
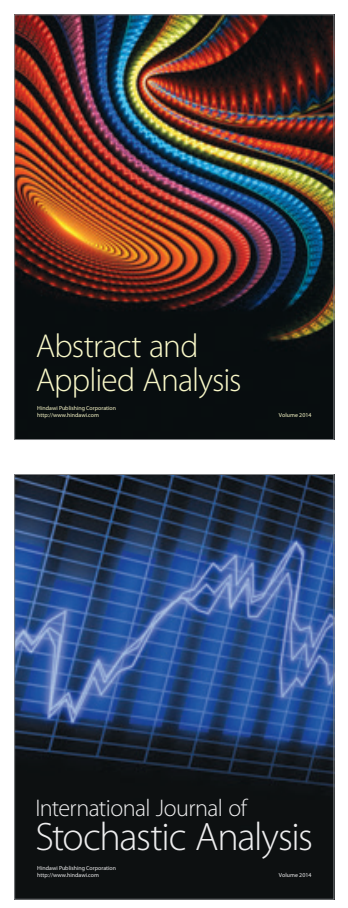

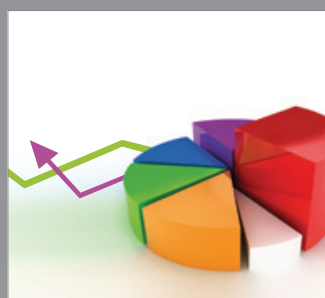

ournal of

Probability and Statistics

Promensencen
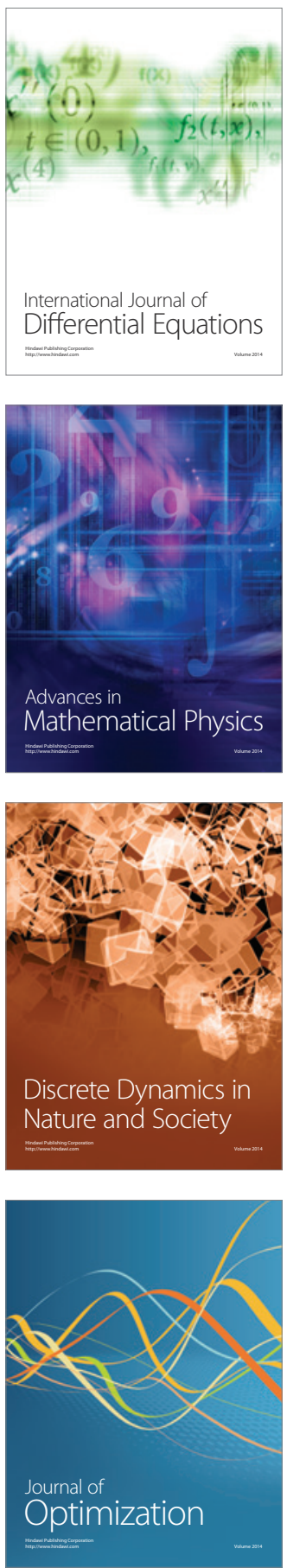